\title{
Identifying sexual differentiation genes that affect Drosophila life
}

\section{span}

\author{
Jie Shen, Daniel Ford, Gary N Landis and John Tower*
}

Address: Molecular and Computational Biology Program, Department of Biological Sciences, University of Southern California, Los Angeles, CA 90089-2910, USA

Email: Jie Shen - jies@usc.edu; Daniel Ford - danielfo@usc.edu; Gary N Landis - landis@usc.edu; John Tower* - jtower@usc.edu

* Corresponding author

Published: 9 December 2009

BMC Geriatrics 2009, 9:56 doi:10.1/86/|47|-23|8-9-56
Received: 15 June 2009

Accepted: 9 December 2009

This article is available from: http://www.biomedcentral.com/l47I-23/8/9/56

(c) 2009 Shen et al; licensee BioMed Central Ltd.

This is an Open Access article distributed under the terms of the Creative Commons Attribution License (http://creativecommons.org/licenses/by/2.0), which permits unrestricted use, distribution, and reproduction in any medium, provided the original work is properly cited.

\begin{abstract}
Background: Sexual differentiation often has significant effects on life span and aging phenotypes. For example, males and females of several species have different life spans, and genetic and environmental manipulations that affect life span often have different magnitude of effect in males versus females. Moreover, the presence of a differentiated germ-line has been shown to affect life span in several species, including Drosophila and C. elegans.
\end{abstract}

Methods: Experiments were conducted to determine how alterations in sexual differentiation gene activity might affect the life span of Drosophila melanogaster. Drosophila females heterozygous for the tudor [I] mutation produce normal offspring, while their homozygous sisters produce offspring that lack a germ line. To identify additional sexual differentiation genes that might affect life span, the conditional transgenic system Geneswitch was employed, whereby feeding adult flies or developing larvae the drug RU486 causes the over-expression of selected UAS-transgenes.

Results: In this study germ-line ablation caused by the maternal tudor[ 1$]$ mutation was examined in a longlived genetic background, and was found to increase life span in males but not in females, consistent with previous reports. Fitting the data to a Gompertz-Makeham model indicated that the maternal tudor [I] mutation increases the life span of male progeny by decreasing age-independent mortality. The Geneswitch system was used to screen through several UAS-type and EP-type P element mutations in genes that regulate sexual differentiation, to determine if additional sex-specific effects on life span would be obtained. Conditional over-expression of transformer female isoform (traf) during development produced male adults with inhibited sexual differentiation, however this caused no significant change in life span. Overexpression of doublesex female isoform ( $d s x F)$ during development was lethal to males, and produced a limited number of female escapers, whereas over-expression of $d s x F$ specifically in adults greatly reduced both male and female life span. Similarly, over-expression of fruitless male isoform A (fru-MA) during development was lethal to both males and females, whereas over-expression of fru-MA in adults greatly reduced both male and female life span.

Conclusion: Manipulation of sexual differentiation gene expression specifically in the adult, after morphological sexual differentiation is complete, was still able to affect life span. In addition, by manipulating gene expression during development, it was possible to significantly alter morphological sexual differentiation without a significant effect on adult life span. The data demonstrate that manipulation of sexual differentiation pathway genes either during development or in adults can affect adult life span. 


\section{Background}

The disposable soma theory suggests that aging occurs because there is a selection pressure to assign limited biological resources to short-term survival, growth, and reproduction, rather than long-term survival $[1,2]$. Consistent with this idea, several studies have suggested that there may exist a trade-off between reproduction and life span. For example, in humans, longer life span has been correlated with a smaller number of offspring [3], but see also $[4,5]$. In C. elegans, elimination of reproduction by ablation of the germ line extended life span by up to $+60 \%[6]$. This effect was attributed to increased activity of the insulin/IGF1-like signaling (IIS) pathway target transcription factor DAF-16 in the gastrointestinal tract, caused by reduced hormonal signaling from the gonad to the intestine [7].

A trade-off between life span and reproduction does not appear to be obligatory, because it is possible in certain instances to increase life span in C. elegans and Drosophila without causing a decrease in reproduction $[8,9]$. One possibility is that it is sexual differentiation, rather than reproduction per se, that exerts a cost on life span [10].

In Drosophila males and females often respond differently to genetic or environmental interventions that extend life span. For example, reduced IIS, dietary restriction and virginity each caused greater increase in life span in females than in males [11,12]. Similarly, over-expression of mutant forms of $p 53$ preferentially increased female life span $[13,14]$. Possible mechanisms for sex-specific life spans include asymmetric inheritance of sex chromosomes, genetic differences between the sexes, differences in physiology and behavior, maternal effects, and sexual selection, sexual conflict, or sex linkage $[10,11,15]$.

In Drosophila, elimination of germ cells (GCs) by forced expression of the differentiation gene bam in late development or adulthood was found to increase median life span by $+14 \%$ to $+78 \%$ in males, and by $+23 \%$ to $+100 \%$ in females [16]. The elimination of Drosophila GCs was found to modulate IIS, by increasing nuclear localization of the Drosophila homolog of DAF-16 (called dFoxo), and by increasing the expression of Drosophila insulin-like peptides (dilps) [16]. The Drosophila maternal effect genes germ cell-less and tudor are necessary for the formation of the germ line in offspring [17]. Interestingly, in another recent study, it was concluded that elimination of Drosophila GCs using germ cell-less and tudor mutations might not extend life span [18], and the reason for the difference in results in these previous studies may be differences in the timing of germ cell ablation relative to fly development. Here the maternal tudor [1] mutation was tested in a particularly long-lived genetic background, and was found to increase the life span of male offspring, but to have neutral or negative effects on female life span.

In the Drosophila soma, sexual differentiation is determined by the $X$ chromosome to autosome (X:A) ratio and the on/off status of the master regulatory gene Sex-lethal $(S x l)$ (Figure 1A), however cell-cell interactions also play a role [19]. Sexual differentiation in the Drosophila germ line is determined by germ line-autonomous signals, as well as the interactions between the germ line and the soma [20-22]. Genes in the somatic sex determination hierarchy play important roles but are not the primary regulators in germ line sexual differentiation. For example, $S x l$ promotes oogenic development in female germ cells, but not in male germ cells.

To test if additional alterations in sexual differentiation might affect fly life span, the Geneswitch system was used to screen through several UAS-type and EP-type P element mutations in genes that regulate sexual differentiation. This approach allows genes to be over-expressed either during Drosophila larval development, or specifically in the adult stages. The Drosophila sex determination hierarchy consists of pre-mRNA splicing factors encoded by the genes Sex-lethal (Sxl), transformer (tra), and transformer-2 (tra-2) [23] (Figure 1A). In females (sex chromosome composition $X / X$ ), the ratio of $X$ chromosomes to autosomes results in the production of SXL protein, which directs the pre-mRNA splicing of tra transcripts. The TRA and TRA-2 proteins together direct the splicing of premRNAs for the transcription factor genes doublesex $(d s x)$ and fruitless (fru), such that females produce the female form of the doublesex protein (DSXF), and no sex-specific forms of Fruitless protein. In males (sex chromosome composition $X / Y$ ), the SXL and TRA products are not produced, which results in production of the male form of doublesex $\left(\mathrm{DSX}^{\mathrm{M}}\right)$, and the male form of Fruitless protein $\left(\right.$ FRU $\left.^{\mathrm{M}}\right)$. The DSX and FRU transcription factors then direct sex-specific differentiation of tissues and the potential for sex-specific behaviors. The data presented here demonstrate that the Drosophila sexual differentiation pathway can act during development and in adults to affect longevity.

\section{Methods \\ Drosophila Strains}

The UAS-transgene strains were generated by various laboratories, and stocks were obtained from Michelle Arbeitman at USC: The strain $y w ; P\{U A S-f r u M A\}[7] ;+$ is described in [24]. The strain $w ; P\{U A S-f r u-I R\} / C \gamma O$; $P\{U A S-f r u-I R\}$ is described in [25]. The strain $w ; P\{U A S-$ $d s x M\} / C y O, G F P ;+$ is described in [26]. The strain $w ;+$; $P\{U A S-d s x F\} / T M 3, S b$ was generated by Ken Burtis and is described in [27]. The strain $w$; P\{UAS-tra $\}[20 J 7] ;+$ is 


\section{A The Drosophila Sex Determination Hierarchy}
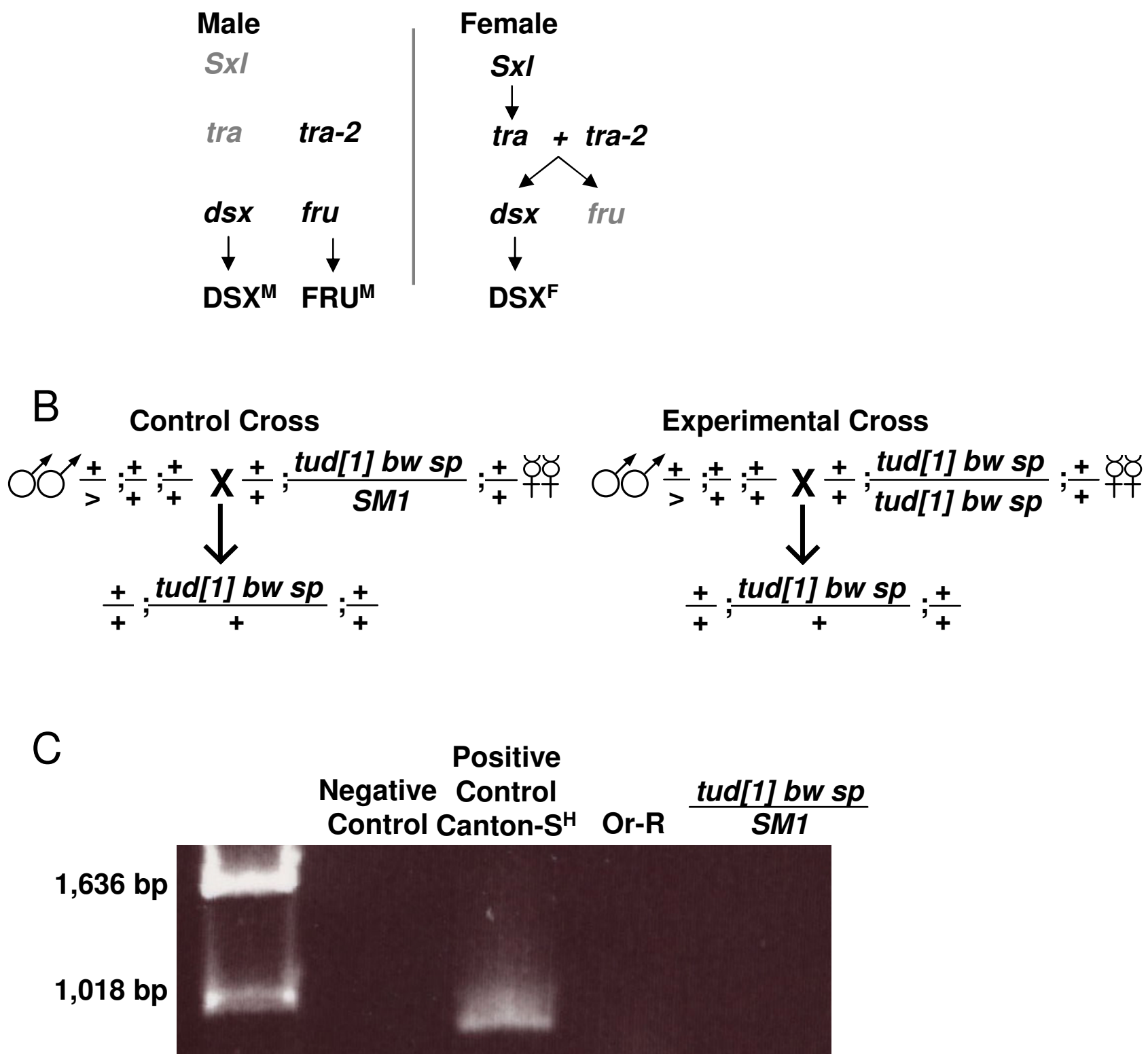

Figure I

Drosophila sex determination hierarchy and the tudor mutation. A) The Drosophila somatic sex determination hierarchy. The $X$ to autosome ratio causes $S x l$ to be "off" in males (indicated in gray color) and to be "on" in females. In males, lack of SXL and TRA activity leads to expression of FRUM and DSXM. In females, TRA and TRA2 together direct splicing of downstream targets to produce DSXF, and fru PI transcripts that are not translated in females. B) Crossing scheme to produce germ line-ablated flies using tudor[I]. For the experimental group, tudor homozygote females were crossed to wild type Oregon- $\mathrm{R}$ males to generate progeny lacking a germ line. For the control group, tudor heterozygote females were crossed to Oregon- $R$ males to generate progeny containing a normal germ line. Both experimental and control groups have the same chromosomal composition. B) Test for Wolbachia. The Wolbachia I6S rDNA sequences were amplified by PCR from the indicated Drosophila lines and controls, and the presence or absence of Wolbachia-specific PCR products was determined by gel electrophoresis and ethidium bromide staining. 
described in [28]. The strains $w ;+; P\{d s x-X P\}$ [d09625] and $w ; P\{$ tra2-XP $\}$ [d10032]; + are described in [29]. The strain $y$ [1] $w$ [67c23] P\{EPgy2\}Sxl [EY06108]/FM6B was obtained from Bloomington Drosophila stock center. The tudor[1] mutant strain [30] was also obtained from Bloomington Drosophila stock center. The genotypes of all strains are presented in Table 1. For experiments involving tudor [1], the experimental group consisted of the progeny of tudor[1] homozygous females crossed to Oregon $\mathrm{R}$ wild-types males, while the control group consisted of the offspring of tudor[1] heterozygotes crossed to Oregon $\mathrm{R}$ males. This resulted in control and experimental groups with the same chromosomal composition, however the experimental group lacks a germ line due to the maternal effect of homozygous tudor [1] (Figure 1B). The tissue-general Geneswitch driver line Act-GS-255B contains multiple copies of a construct in which the tissue-general actin $5 \mathrm{C}$ promoter drives expression of the Geneswitch protein, and has previously been described and characterized $[13,31]$. Act-GS-255B virgins were used in the crosses with males of other lines, unless the UAS insertion of the sex differentiation gene or the EP (XP) insertion was on the $X$ chromosome, in which case the cross direction was reversed.

\section{Fly Culture}

Drosophila culture and life span assays were performed essentially as previously described [31]. Briefly, Drosophila were cultured on a dextrose, agar, yeast, and cornmeal media [32], and adults were maintained at twenty-five flies per vial. Survival assays were performed at $25^{\circ} \mathrm{C}$. Every two days, flies were transferred to new vials, and the number of deaths was recorded. The drug RU486 (Mifepristone, Sigma) was dissolved in ethanol $(100 \%)$ to make a stock solution at $3.2 \mathrm{mg} / \mathrm{ml}$. For adult feeding, 50 ul of RU486 stock solution was added to each vial to produce a final concentration of $\sim 160 \mathrm{ug} / \mathrm{ml} ; 50$ ul ethanol was added to the control vials. For larval feeding, $0.5 \mathrm{ml}$ of $3.2 \mathrm{mg} / \mathrm{ml}$ RU486 stock solution was added to each bottle, whereas $0.5 \mathrm{ml}$ ethanol was added to controls. Vials and bottles were covered with cheesecloth and allowed to dry overnight to allow the ethanol to evaporate.

\section{Wolbachia Test}

Total DNA was extracted from ten male and ten female flies of each line using the ZR Genomic DNA Kit II (Zymo Research). The Drosophila DNA was then used as template for PCR amplification with Wolbachia-specific primers, and the products were fractionated on an agarose gel and stained with ethidium bromide $[32,33]$.

\section{Phenotype Characterization}

UAS-tra males were crossed to Act-GS-255B virgins, and the progeny were cultured on food with drug (+RU486) to drive the over expression of tra during development, or cultured on food with ethanol as the control. Male and female external genitalia, abdomenal pigmentation patterns, and male sex combs were photographed using a Leica MZ FLIII fluorescence stereomicroscope. Or-R and $w$ [1118] males were also crossed to Act-GS-255B virgins, and the progeny were cultured on food supplemented with drug (+RU486) or with ethanol only, as the controls.

\section{Statistical Analysis}

Mean, standard deviation, median, percent change in mean, percent change in median, and log rank $p$ value were calculated using R 2.6.2. Analysis of mortality rate was performed using the WinModest statistical package [34]. The best fit model was determined by the "Model Comparison" option and confirmed by other additional options of WinModest software. In the Gompertz-Makeham model, the increase of mortality $\left(\mu_{x}\right)$ with age $(x)$ is expressed as: $\mu_{x}=a e^{b x}+c$, where the constant $a$ is the initial

Table I: Starting stocks.

\begin{tabular}{|c|c|c|c|}
\hline St\# & Genotype & Notes & Abbreviation \\
\hline I & w [IIII 8 ]; Act-GS-255B;+ & Tissue-general Geneswitch driver & $255 B$ \\
\hline 2 & y w; P\{UAS-fruMA\}[7]; + & UAS-fru male $A$ isoform & fruMA \\
\hline 3 & $w ; P\{U A S-f r u-I R\} / C y O ; P\{U A S-f r u-I R\}$ & UAS-fru RNAi & frulR \\
\hline 4 & w; P\{UAS-dsxM\}/CyO, GFP; + & UAS-dsx male isoform & $d s \times M$ \\
\hline 5 & w P $\{$ UAS-tra2-IR\} [6IA]; +; P\{UAS-tra2-IR\} [82A] & UAS-tra2 RNAi & $\operatorname{tra} 2 I R$ \\
\hline 6 & $w[1 / 18] ;+;+$ & Injection strain control & \\
\hline 7 & Oregon $R(+;+;+)$ & wild type control & \\
\hline 9 & $w ;+; P\{U A S-d s x F\} / T M 3, S b$ & UAS-dsx female isoform & $d s x$ \\
\hline 10 & w; P\{UAS-tra $\}$ [20J7]; + & UAS-tra & tra \\
\hline II & $y[1] w[67 c 23] P\{E P g y 2\} S x I[E Y 06 / 08] / F M 6 B$ & $E P$-sxl & $s x l$ \\
\hline 14 & $w ;+; P\{d s x-X P\}[d 09625]$ & $X P-d s X$ & $d s x$ \\
\hline 15 & w; $P\{\operatorname{tra2}-X P\}[d / 0032] ;+$ & $X P$-tra2 & tra2 \\
\hline 16 & $+;$ tud [I] bw sp/SMI; + & tudor[I] mutant & \\
\hline
\end{tabular}


mortality rate, $b$ is the rate of exponential increase in mortality, and $\mathrm{c}$ is the age-independent mortality. WinModest assumes that the observation on the cohorts is continuous and ages at death are exact. Fly deaths were recorded every other day. Therefore, age was divided by two before being input into WinModest. The output age was then multiplied by two to compensate for the initial division. Without dividing the age by two and then multiplying the output by two, the trend-lines based on the parameters $(\mathrm{a}, \mathrm{b}, \mathrm{c})$ would be too low, and would not perfectly match the mortality data points. The natural logarithm of mortality $\left(\operatorname{Ln}\left(\mu_{x}\right)\right)$ for each time point was calculated by the WinModest statistical software, based on: $\mathrm{P}_{x}=\mathrm{N}_{x+1} / \mathrm{N}_{x}, \mu_{x}=$ $\operatorname{Ln}\left(\mathrm{P}_{x}\right)$. Parameters $(\mathrm{a}, \mathrm{b}, \mathrm{c})$ were also calculated by the WinModest statistical package, based on a likelihood ratio test. The full model $\left(a e^{b x}+c\right)$ was plotted, and the Gompertz-only component $\left(\mu_{x}\right)$ was used to build the decomposed survival curves, from the reverse calculation of $\mu_{x}$ : $\mu_{x}=a \mathrm{e}^{b x}, \mathrm{P}_{x}=\mathrm{e}^{-\mu_{x}}$. For the decomposed survival curves, any value below $0.5 \%$ survival was considered to be the final data point.

\section{Results}

Female flies heterozygous or homozygous for the tudor [1] mutation were crossed to Oregon-R wild-type male flies to produce control offspring and offspring lacking the germ line, respectively (Figure 1B). In the first experiment the life span of control and germ line-ablated flies was measured using cohorts of $\sim 125$ flies each. For the germ lineablated male flies the mean and median life span was increased by $+19.9 \%$ and $+12.9 \%$, respectively, relative to controls, whereas in contrast female life span was significantly decreased (Table 2). To determine if these results were reproducible, the experiment was repeated with cohorts sizes of $\sim 240$ flies. Male mean and median life span was again found to be increased by $+19.97 \%$ and
$+12.2 \%$, respectively, whereas female life span was not altered. Plots of percent survival versus time indicated that there was also significant early mortality in several of the cohorts (Figure 2A, D, G, J). Consequently, the Winmodest statistical package was used to control for early mortality and to analyze the survival data in greater detail. The data was fitted to a Gompertz-Makeham model where the constant $a$ is the initial mortality rate, $b$ is the rate of exponential increase in mortality, and $\mathrm{c}$ is the age-independent mortality (Figure 2B, E, H, K). The values of $\mathrm{a}, \mathrm{b}$ and $\mathrm{c}$ were calculated based on a likelihood ratio test (Table 3 ). Replotting of the fitted data using only the Gompertz term yields a decomposed survival curve consisting of only the age-dependent mortality (Figure 2C, F, I, L). The data indicate that the increase in mean life span of germ lineablated males relative to controls can be attributed to a decrease in age-independent mortality (rate constant $c$ ), while the initial mortality rate (rate constant $a$ ) and mortality rate increase with time (rate constant $b$ ) were not significantly affected (Table 3 ). While the initial experiment indicated a decrease in the life span of germ line-ablated females relative to controls, this result was not reproduced in the larger cohorts, where there was no significant difference in any of the mortality rate parameters between germ line-ablated females and controls.

Wolbachia are gram-positive bacteria that are transmitted in Drosophila through maternal inheritance [35]. These bacteria are capable of altering life span, and can potentially result in a false positive for life span extension $[8,36]$. Therefore, the presence of Wolbachia was assayed by PCR using primers specific for the Wolbachia 16S RNA genes, and the lines used in this experiment were found not to be infected (Figure 1C).

To begin to ask if other alterations in sexual differentiation could affect life span, the Geneswitch system was used to cause over-expression or inhibition of several genes involved in the sex-determination pathway. Expression of the Geneswitch transcription factor was driven

Table 2: Statistical analysis of tudor life span assays.

\begin{tabular}{|c|c|c|c|c|c|c|}
\hline Group & $\mathbf{N}$ & Mean $^{a}$ & Median & $\%$ Change in Mean & \%Change in Median & Log Rank p Value \\
\hline \multicolumn{7}{|l|}{$\operatorname{Exp} 1$} \\
\hline Mutant Males & 125 & $68.45 \pm 11.37$ & 70 & 19.91 & 12.90 & 4.25E-05 \\
\hline Control Males & 122 & $57.08 \pm 20.28$ & 62 & & & \\
\hline Mutant Females & 118 & $47.36 \pm 24.50$ & 56 & -20.99 & -23.29 & 5.33E-IO \\
\hline Control Females & 124 & $59.94 \pm 28.03$ & 73 & & & \\
\hline \multicolumn{7}{|l|}{$\operatorname{Exp} 2$} \\
\hline Mutant Males & 238 & $87.41 \pm 18.03$ & 92 & 19.97 & 12.20 & I.00E- 10 \\
\hline Control Males & 139 & $72.86 \pm 25.01$ & 82 & & & \\
\hline Mutant Females & 233 & $74.33 \pm 22.01$ & 80 & -2.04 & 0.00 & 0.121 \\
\hline Control Females & 175 & $75.87 \pm 21.78$ & 80 & & & \\
\hline
\end{tabular}

a Mean life span, days +/- SD. 
Table 3: Parameters for Gompertz-Makeham model and likelihood ratio test results.

\begin{tabular}{|c|c|c|c|c|c|c|c|c|c|}
\hline & Parameters & Mutant & Control & chi $^{2}$ & df & p Value & chi $^{2}$ & df & p Value \\
\hline Tudor $\operatorname{Exp} I$ & & \multicolumn{5}{|c|}{ one parameter compared at each time } & & & \\
\hline \multirow[t]{4}{*}{ Males } & & & & & & & \multicolumn{3}{|c|}{ Both $\mathrm{a}$ and $\mathrm{b}$ are constrained } \\
\hline & $\mathrm{a}$ & $1.00 \times 10^{-4}$ & $7.00 \times 10^{-5}$ & 0.13 & I & 0.724 & & & \\
\hline & $\mathrm{b}$ & $2.07 \times 10^{-1}$ & $2.29 \times 10^{-1}$ & 0.73 & i & 0.392 & & & \\
\hline & c & $2.06 \times 10^{-9}$ & $7.52 \times 10^{-3}$ & 17.65 & I & $<0.001$ & 23.76 & 1 & $<0.001$ \\
\hline \multirow[t]{4}{*}{ Females } & & & & & & & \multicolumn{3}{|c|}{$\mathrm{c}$ is constrained } \\
\hline & $\mathrm{a}$ & $3.20 \times 10^{-4}$ & $1.04 \times 10^{-6}$ & 13.23 & I & $<0.001$ & 13.34 & 1 & $<0.001$ \\
\hline & $\mathrm{b}$ & $1.93 \times 10^{-1}$ & $2.91 \times 10^{-1}$ & 5.84 & I & 0.016 & 5.47 & 1 & 0.019 \\
\hline & c & $1.43 \times 10^{-2}$ & $1.66 \times 10^{-2}$ & 0.37 & I & $0.54 I$ & & & \\
\hline \multicolumn{10}{|l|}{ Tudor $\operatorname{Exp} 2$} \\
\hline \multirow[t]{4}{*}{ Males } & & & & & & & \multicolumn{3}{|c|}{ Both $\mathrm{a}$ and $\mathrm{b}$ are constrained } \\
\hline & $\mathrm{a}$ & $8.38 \times 10^{-8}$ & $6.03 \times 10^{-7}$ & 2.60 & I & 0.107 & & & \\
\hline & $b$ & $3.19 \times 10^{-1}$ & $3.06 \times 10^{-1}$ & 0.23 & I & 0.633 & & & \\
\hline & c & $1.67 \times 10^{-3}$ & $4.67 \times 10^{-3}$ & 7.98 & I & 0.005 & 18.46 & 1 & $<0.001$ \\
\hline \multicolumn{10}{|l|}{ Females } \\
\hline & $\mathrm{a}$ & $6.40 \times 10^{-7}$ & $3.46 \times 10^{-7}$ & 0.33 & I & 0.565 & & & \\
\hline & $b$ & $3.07 \times 10^{-1}$ & $3.17 \times 10^{-1}$ & 0.15 & I & 0.694 & & & \\
\hline & c & $3.76 \times 10^{-3}$ & $3.69 \times 10^{-3}$ & 0.00 & I & 0.949 & & & \\
\hline
\end{tabular}

with the cytoplasmic actin Actin5C promoter, using transgenic line Act-GS-255B. Feeding animals the drug RU486/ Mifepristone either during larval development or as adults causes activation of the Geneswitch transcription factor, which then binds to UAS sites in target promoters and activates expression of the gene of interest or the RNAi construct. To control for any effects of the drug itself, the Act-GS-255B line was crossed to Oregon R wild type strain to generate progeny containing Act-GS-255B but no target construct. In these control flies, the drug treatment generally had no effect on life span, with the exception of two experiments where life span of adult females was reduced by $-2 \%$ to $-5 \%$ (Figure 3A, B; Table 4 ). The Act-GS-255B line was also crossed to the $w$ [1118] injection strain to generate another control. In these control flies, the drug treatment had no effect on male life span, and had a slight effect on female life span ranging from $-5 \%$ to $+5 \%$ (Table 4). However, because these changes in controls were small in magnitude and were not always observed, we interpret these changes as being within the background of the assay. The drug treatment during development caused no visible alterations in sexual differentiation of the flies (data not shown).

Conditional over-expression of the pre-mRNA splicing factor gene tra during development significantly inhibited sexual differentiation in males, resulting in a lack of much of the external genitalia and a reduction in the size of the sex combs (Figure 4C, D, E, F), however these flies did not exhibit a significant change in life span (Figure 3C; Table 4). In contrast, in females, over-expression of tra during development did not detectably affect sexual differentiation (Figure 4A, B), and did not alter life span (Figure 3D; Table 4). Over-expression of tra in adults gave no signifi- cant change in male and female life span (Figure 3C, D; Table 4). Repeats of the life span assay with tra overexpression produced similar results (Table 4, Exp3).

Conditional expression of the tra2 RNAi construct in female adults resulted in a small but significant decrease in life span of $-12 \%$ (Figure 3F). However, expression of the tra2 RNAi construct during male and female development or in male adults did not affect life span (Figure 3E, F; Table 4), nor did it have a detectable effect on morphological differentiation.

Over-expression of $d s x M$ during development was toxic to males and females and produced only a limited number of adult escapers, while over-expression of $d s x \mathrm{M}$ in adults caused a small but statistically significant reduction in male and female life span, $-5.5 \%$ and $-7.8 \%$, respectively (Figure 3G, H and Table 4 Exp1); however, this apparent reduction in life span upon over-expression of $d s x \mathrm{M}$ in adults may not be significant, as it was similar in magnitude to the small variation in life span observed in controls.

Over-expression of $d s x F$ during development was lethal to males and produced a limited number of females. This observation is consistent with previous studies where over-expression of DSX during development using a heat shock promoter was found to cause lethality in both males and females [37]. Interestingly, over-expression of $d s x F$ specifically in adults dramatically reduced male and female mean life span, by $-61.3 \%$ and $-45.8 \%$, respectively (Figure 3I, J; Table 4, Exp1); and these results were confirmed by repeated experiments (Table 4, Exp3). Similarly, over-expression of the fru male isoform A (fru MA) 

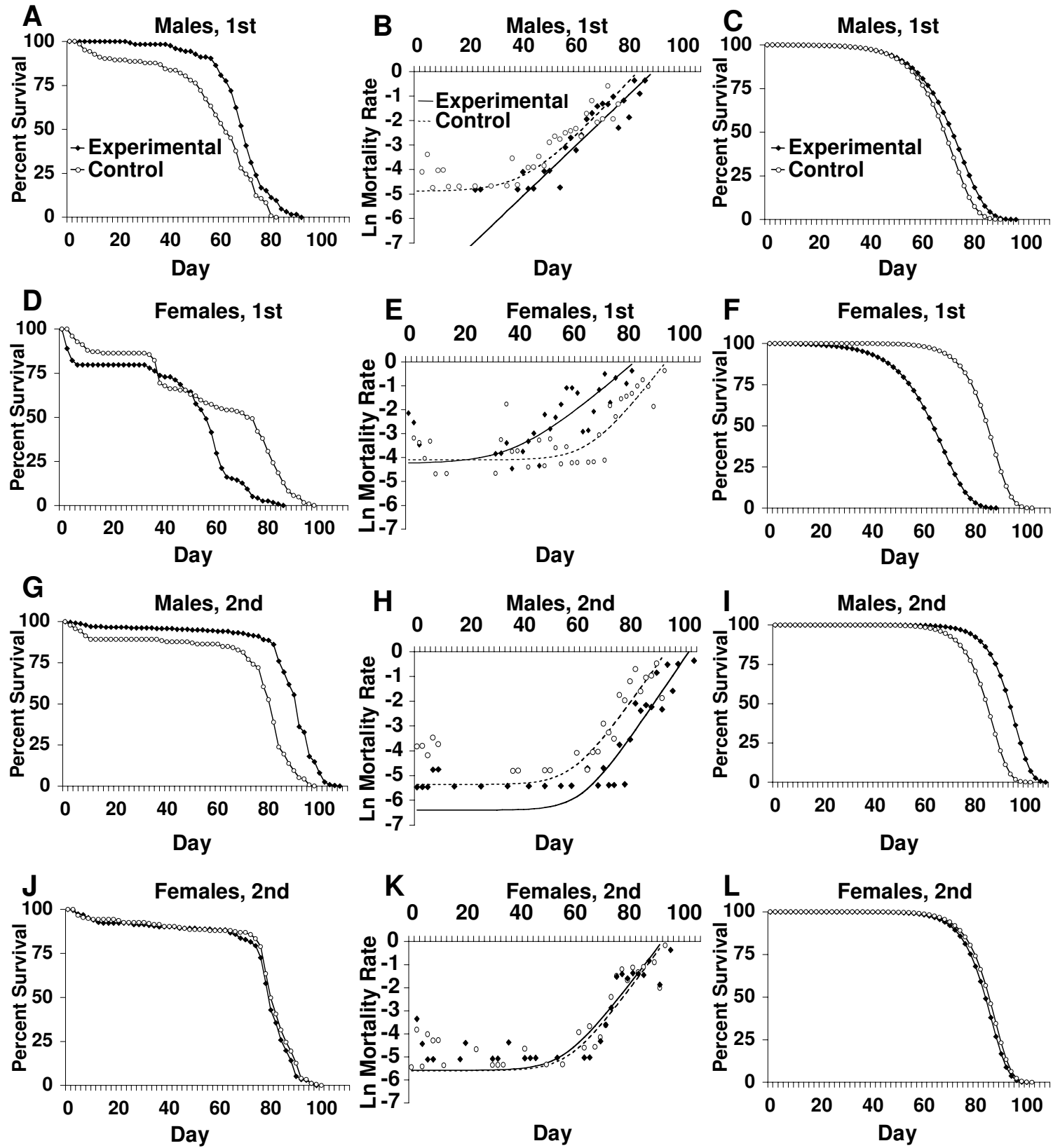

\section{Figure 2}

Life span assays and mortality rate analysis for germ line-ablated Drosophila. A) Males lacking the germ line. The assay consisted of 125 flies for the experimental group and 122 flies for the control group. B) Mortality rate (Gompertz-Makeham model) of the germ line-ablated and control male groups. C) Redrawn survival curve for males with age-independent mortality removed. D) Females lacking the germ line. The assay consisted of I I 8 flies for the experimental group, and I 24 flies for the control group. E) Mortality rate (Gompertz-Makeham model) of the germ line-ablated and control female groups. F)

Redrawn survival curve for females with age-independent mortality removed. G) Males lacking the germ line, repeat assay. The assay consisted of 238 flies for the experimental group and 139 flies for the control group. H) Mortality rate (Gompertz-Makeham model) of the germ line-ablated and control male groups. I) Redrawn survival curve for males with age-independent mortality removed. J) Females lacking the germ line, repeat assay. The assay consisted of 233 flies for the experimental group and I 75 flies for the control group. K) Mortality rate (Gompertz-Makeham model) of the germ line-ablated and control female groups. L) Redrawn survival curve with age-independent mortality removed. 
during development was lethal, and over-expression of fru $M A$ in adults greatly reduced both male and female mean life span, by $-61.97 \%$ and $-64.5 \%$, respectively (Figure $3 \mathrm{~K}$, L; Table 4, Exp1).

Developmental or adult-specific expression of one or two inserts of an RNAi construct designed to target the malespecific isoform of FRU [25] did not affect male life span (Figure 3M), but surprisingly, decreased female lifespan significantly (Figure $3 \mathrm{~N}$; Table 4 ). Previous studies where the Act-GS-255B driver and various RNAi constructs were used to inactive expression of several genes demonstrates that RNAi per se does not affect life span $[38,39]$.

Finally, EP-sxl, XP- $d s x$, and XP-tra2 gene-over-expression lines were also tested, but did not give a consistent or significant change in life span ( $<3 \%$ change), when expressed during development or in adults (Table 4, Exp3).

\section{Discussion}

In these experiments, germ line ablation using the tudor[1] mutation was examined in a long-lived genetic background, and was found to cause increased mean and median life span in males $(+19 \%$ and $+12 \%$, respectively), whereas female life span was not affected. In a previous report, germ line ablation using the tudor[1] mutation increased the median life span of male flies by $+8.6 \%$ [18]. However, germ line ablation was considered not to have increased life span in that report. Presumably, this was due to a decrease in female life span, and the fact that germ line ablation with a germ cell-less mutant failed to extend life span in either males or females. However, germ cell-less was reported to have only $\sim 75 \%$ penetrance in that study, which conceivably could have masked any life span extension. Here the tudor mutation was found to show a greater increase in life span, and overall longer life spans were observed for both control and experimental groups. This is likely due to differences in the genetic background employed: here the relatively long-lived Oregon-R strain was used for crosses to tudor [1], and the control males and control females had median life spans of 82 days and 80 days, respectively (Table 2); whereas in the previous study the relatively shorter-lived Dahomey strain was utilized for crosses, and produced male control median life span of 60 days, and female control median life span of 71 days. In a previous study of D. subobscura, "ovariless" females lived significantly longer than virgin female controls or mated female controls [40], and the difference between this and the present results may be due to the difference in genetic background, or the difference in the way or extent of germ line removal caused by specific mutations. In another recent report, elimination of GCs was found to extend median life span by $+14 \%$ to $+78 \%$ in males, and by $+23 \%$ to $+100 \%$ in females [16]
In those experiments germ line ablation was produced by mis-expression of the bag of marbles (bam) gene in adult flies, which caused full sterility by day 7 post-eclosion in females, and GC depopulation in the $3^{\text {rd }}$ instar larval (L3) stage or later in males. The presence of a complete germ line in the earlier stages of development followed by GC loss at a later stage could affect life span in a different way, compared with the lack of a germ line from the beginning of embryogenesis, such as is produced by the tudor mutation. For example, the tudor mutation also partially inhibits formation of the somatic gonad, while ablation of GCs using bam mis-expression at later developmental stages allows for complete differentiation of the somatic gonad. Consistent with this idea, in C. elegans, extension of life span by ablation of the germ line requires the presence of the somatic gonad [41].

The Winmodest program was used to fit the tudor[1] mutant data to a Gompertz-Makeham model, which allowed us to separate early mortality from the agedependent and age-independent mortality. The analysis indicated that germ line ablation in male Drosophila extended life span by decreasing the age-independent mortality. That implies that germ line ablation provides a benefit for survival in male flies, and this beneficial effect is constant over the adult life span. This might occur through altered IIS as reported for $C$. elegans hermaphrodites [41].

The other manipulations of the sexual differentiation pathway tested produced either neutral or negative effects on adult fly life span. For example, conditional overexpression of tra during development significantly inhibited sexual differentiation in males, yet produced no significant change in adult life span. Expression of tra in males has previously been found to feminize locomotor activity patterns [42]. These data demonstrate that it is possible to alter sexual differentiation and perhaps even behavior, at least in males, without necessarily having effects on adult life span.

Over-expression of $d s x F$ during development was lethal to males and produced a limited number of females, while over-expression of $d s x F$ in adults greatly reduced both male and female life span. Over-expression of $d s x M$ during development was toxic to males and females, whereas over-expression of $d s x \mathrm{M}$ in adults produced only a small, if any, reduction in male and female life span. This indicates that in adults, where sexual differentiation is already complete, changes in expression of specific sex-determination pathway genes are still able to have significant effects on life span.

Interestingly, over-expression of fru male isoform A ( fruMA) during development was lethal, and over-expres- 
Table 4: Life span data with means, standard deviations, medians, percent change in mean and median, and log rank $p$ value.

\begin{tabular}{|c|c|c|c|c|c|c|c|c|c|}
\hline $\begin{array}{l}\text { Cross } \\
\text { MxF }\end{array}$ & RU486 & Genotype & Sex & $\mathbf{N}$ & Mean $^{a}$ & Median & $\begin{array}{l}\text { \%Change in } \\
\text { Mean }\end{array}$ & $\begin{array}{l}\text { \%Change in } \\
\text { Median }\end{array}$ & $\begin{array}{l}\text { Log } \\
\text { Rank } \\
\text { p Value }\end{array}$ \\
\hline \multicolumn{10}{|l|}{$\operatorname{Exp} I$} \\
\hline $7-1$ & - & $w / Y ; 255 B /+;+$ & $M$ & 122 & $68.85 \pm 14.18$ & 71 & --------- & --------- & --------- \\
\hline $7-1$ & $A$ & $w / Y ; 255 B /+;+$ & $M$ & 112 & $70.64 \pm 13.48$ & 73 & 2.6 & 2.82 & 0.646 \\
\hline $7-1$ & $\mathrm{~L}$ & $w / Y ; 255 B /+;+$ & $M$ & 145 & $69.63 \pm 12.16$ & 73 & 1.14 & 2.82 & 0.265 \\
\hline $7-1$ & - & $w /+; 255 B /+;+$ & $\mathrm{F}$ & 121 & $87.78 \pm 8.83$ & 89 & --------- & --------- & -------- \\
\hline $7-1$ & $A$ & $w /+; 255 B /+;+$ & $\mathrm{F}$ & 117 & $88.56 \pm 6.85$ & 89 & 0.89 & 0 & 0.615 \\
\hline $7-1$ & $\mathrm{~L}$ & $w /+; 255 B /+;+$ & $F$ & 120 & $85.96 \pm 11.95$ & 89 & -2.07 & 0 & 0.192 \\
\hline $10-1$ & - & w/Y; 255B/tra; + & $M$ & 122 & $68.60 \pm 15.83$ & 71 & --------- & -------- & -------- \\
\hline $10-1$ & $A$ & w/Y; 255B/tra; + & $M$ & 118 & $73.10 \pm 13.38$ & 73 & 6.56 & 2.82 & 0.024 \\
\hline $10-1$ & $\mathrm{~L}$ & w/Y; 255B/tra; + & $M$ & 123 & $67.54 \pm 14.05$ & 69 & -1.54 & -2.82 & 0.406 \\
\hline $10-1$ & - & $w ; 255 B / t r a ;+$ & $\mathrm{F}$ & 122 & $68.75 \pm 18.34$ & 75 & -------- & -------- & --------- \\
\hline $10-1$ & A & w; 255B/tra; + & $\mathrm{F}$ & 122 & $66.51 \pm 15.22$ & 71 & -3.27 & -5.33 & 4.9 IE-05 \\
\hline $10-1$ & $\mathrm{~L}$ & w; 255B/tra; + & $\mathrm{F}$ & 127 & $73.03 \pm 13.07$ & 77 & 6.22 & 2.67 & 0.15 \\
\hline $\mathrm{I}-5$ & - & w tra2IR/Y;255B/+;tra2IR/+ & $M$ & 119 & $74.82 \pm 13.69$ & 77 & ------- & ------- & -------- \\
\hline $1-5$ & $A$ & w tra2IR/Y;255B/+;tra2IR/+ & M & 120 & $75.33 \pm 12.55$ & 75 & 0.69 & -2.6 & 0.905 \\
\hline$I-5$ & $\mathrm{~L}$ & w tra2IR/Y;255B/+;tra2IR/+ & $M$ & 122 & $76.28 \pm 13.32$ & 81 & 1.96 & 5.19 & 0.628 \\
\hline I-5 & - & w tra2IR/w;255B/+;tra2IR/+ & $\mathrm{F}$ & 119 & $80.43 \pm 22.10$ & 91 & ------- & ------- & ------- \\
\hline $1-5$ & $A$ & w tra2IR/w;255B/+;tra2IR/+ & $\mathrm{F}$ & 128 & $74.20 \pm 19.64$ & 80 & -7.74 & -12.09 & 4.02E-07 \\
\hline$I-5$ & $\mathrm{~L}$ & w tra2IR/w;255B/+,tra2IR/+ & $\mathrm{F}$ & 127 & $82.20 \pm 20.76$ & 89 & 2.21 & -2.2 & 0.218 \\
\hline $4-1$ & - & $w / Y ; 255 B / d s x M ;+$ & M & 120 & $72.39 \pm 12.77$ & 73 & -------- & -------- & -------- \\
\hline $4-1$ & $A$ & $w / Y ; 255 B / d s x M ;+$ & M & 118 & $66.14 \pm 13.80$ & 69 & -8.64 & -5.48 & 2.07E-04 \\
\hline $4-1$ & $\mathrm{~L}$ & $w / Y ; 255 B / d s x M ;+$ & $M$ & $\mathrm{I}$ & $67.00 \pm N A$ & 67 & -7.45 & -8.22 & 0.343 \\
\hline $4-1$ & - & w;255B/dsxM; + & $\mathrm{F}$ & 118 & $77.20 \pm 10.40$ & 77 & --------- & --------- & --------- \\
\hline $4-1$ & $A$ & $w ; 255 B / d s x M ;+$ & $\mathrm{F}$ & 121 & $71.91 \pm 6.83$ & 71 & -6.86 & -7.79 & 6.60E-08 \\
\hline $4-1$ & $\mathrm{~L}$ & w; 255B/dsxM; + & $\mathrm{F}$ & 10 & $69.60 \pm 18.21$ & 70 & -9.85 & -9.09 & 0.765 \\
\hline $9-1$ & - & $w / Y ; 255 B /+; d s x F /+$ & $M$ & 115 & $72.23 \pm 10.20$ & 75 & -------- & --------- & --------- \\
\hline $9-1$ & $A$ & $w / Y ; 255 B /+; d s x F /+$ & $M$ & 108 & $29.78 \pm 7.60$ & 29 & -58.77 & -61.33 & 0 \\
\hline $9-1$ & $\mathrm{~L}$ & $w / Y ; 255 B /+; d s x F /+$ & $M$ & 0 & -------- & --- & -------- & -------- & -------- \\
\hline $9-1$ & - & $w ; 255 B /+; d s x F /+$ & $\mathrm{F}$ & 117 & $77.05 \pm 19.31$ & 83 & --------- & --------- & --------- \\
\hline $9-1$ & $A$ & $w ; 255 B /+; d s x F /+$ & $\mathrm{F}$ & 99 & $44.06 \pm 13.39$ & 45 & -42.82 & -45.78 & 0 \\
\hline $9-1$ & $\mathrm{~L}$ & $w ; 255 B /+; d s x F /+$ & $\mathrm{F}$ & 23 & $71.57 \pm 21.55$ & 73 & -7.12 & -12.05 & 0.191 \\
\hline $2-1$ & - & w/Y; 255B/fruMA; + & $M$ & 118 & $69.27 \pm 12.50$ & 71 & --------- & --------- & --------- \\
\hline $2-1$ & $A$ & $w / Y ; 255 B / f r u M A ;+$ & $M$ & 126 & $27.83 \pm 4.03$ & 27 & -59.83 & -61.97 & 0 \\
\hline $2-1$ & $\mathrm{~L}$ & w/Y; 255B/fruMA; + & M & 0 & --------- & ---- & --------- & --------- & --------- \\
\hline $2-1$ & - & w/y w; 255B/fruMA; + & $\mathrm{F}$ & 113 & $90.34 \pm 10.49$ & 93 & -------- & --------- & --------- \\
\hline $2-1$ & $A$ & w/y w; 255B/fruMA; + & $\mathrm{F}$ & 123 & $32.83 \pm 7.76$ & 33 & -63.66 & -64.52 & 0 \\
\hline $2-1$ & $\mathrm{~L}$ & w/y w; 255B/fruMA; + & $\mathrm{F}$ & 0 & -------- & ---- & --------- & --------- & -------- \\
\hline $3-1$ & - & w/Y; 255B/frulR; frulR/+ & $M$ & 114 & $68.63 \pm 12.39$ & 71 & -------- & -------- & -------- \\
\hline $3-1$ & $A$ & $w / Y ; 255 B / f r u l R ;$ frulR/+ & $M$ & 121 & $68.02 \pm 12.20$ & 69 & -0.88 & -2.82 & 0.77 \\
\hline $3-1$ & $\mathrm{~L}$ & w/Y; 255B/frulR; frulR/+ & $M$ & 124 & $66.23 \pm 13.69$ & 67 & -3.49 & -5.63 & 0.475 \\
\hline $3-1$ & - & w; 255B/frulR; frulR/+ & $\mathrm{F}$ & 116 & $76.37 \pm 16.00$ & 79 & --------- & -------- & -------- \\
\hline $3-1$ & A & w; 255B/frulR; frulR/+ & $\mathrm{F}$ & 120 & $72.94 \pm 11.19$ & 75 & -4.49 & -5.06 & 3.26E-07 \\
\hline $3-1$ & $\mathrm{~L}$ & w; 255B/frulR; frulR/+ & $\mathrm{F}$ & 124 & $63.72 \pm 21.87$ & 65 & -16.57 & -17.72 & 0.002 \\
\hline $6-1$ & - & $w / Y ; 255 B /+;+$ & $M$ & 116 & $77.52 \pm 13.06$ & 78 & ------- & -------- & -------- \\
\hline $6-1$ & $A$ & $w / Y ; 255 B /+;+$ & M & 118 & $77.46 \pm 15.82$ & 79 & -0.08 & 1.28 & 0.125 \\
\hline $6-1$ & $\mathrm{~L}$ & $w / Y ; 255 B /+;+$ & M & 115 & $76.37 \pm 14.50$ & 79 & -1.47 & 1.28 & 0.361 \\
\hline $6-1$ & - & $w / w / I / 8 ; 255 B /+;+$ & $\mathrm{F}$ & 124 & $78.97 \pm 13.48$ & 79 & -------- & -------- & -------- \\
\hline $6-1$ & $A$ & $w / w / I / 8 ; 255 B /+;+$ & $\mathrm{F}$ & 121 & $76.71 \pm 13.57$ & 81 & -2.86 & 2.53 & 0.008 \\
\hline $6-1$ & $\mathrm{~L}$ & $w / w / I / 8 ; 255 B /+;+$ & $\mathrm{F}$ & 123 & $81.28 \pm 14.17$ & 83 & 2.93 & 5.06 & 0.118 \\
\hline \multicolumn{10}{|l|}{$\operatorname{Exp} 2$} \\
\hline $3-1$ & - & w/Y; 255B/frulR; frulR/+ & M & 88 & $48.77 \pm 26.56$ & 58 & -------- & --------- & --------- \\
\hline $3-1$ & $A$ & $w / Y ; 255 B / f r u l R ;$ frulR/+ & $M$ & 109 & $61.43 \pm 16.27$ & 64 & 25.95 & 10.34 & 0.024 \\
\hline $3-1$ & $\mathrm{~L}$ & w/Y; 255B/frulR; frulR/+ & $M$ & 120 & $53.65 \pm 19.56$ & 56 & 10 & -3.45 & 0.895 \\
\hline $3-1$ & - & w; 255B/frulR; frulR/+ & $\mathrm{F}$ & 76 & $68.45 \pm 17.05$ & 73 & -------- & -------- & --------- \\
\hline $3-1$ & $A$ & w; 255B/frulR; frulR/+ & $\mathrm{F}$ & 95 & $58.04 \pm 14.35$ & 62 & -15.2 & -15.07 & I.55E-I 5 \\
\hline $3-1$ & $\mathrm{~L}$ & w; 255B/frulR; frulR/+ & $\mathrm{F}$ & 129 & $53.77 \pm 18.60$ & 60 & -21.45 & $-|7.8|$ & $1.14 E-13$ \\
\hline
\end{tabular}


Table 4: Life span data with means, standard deviations, medians, percent change in mean and median, and log rank p value. (Continued)

\begin{tabular}{|c|c|c|c|c|c|c|c|c|c|}
\hline $3-1$ & - & w/Y; 255B/CyO; frulR/+ & $M$ & 55 & $42.18 \pm 18.86$ & 46 & '------- & $\begin{array}{l}------ \\
---\end{array}$ & $\begin{array}{l}------- \\
--1\end{array}$ \\
\hline $3-1$ & A & $w / Y ; 255 B / C y O ;$ frulR/+ & $M$ & 66 & $41.52 \pm 12.24$ & 44 & -1.58 & -4.35 & 0.066 \\
\hline $3-1$ & $\mathrm{~L}$ & $w / Y ; 255 B / C y O ;$ frulR/+ & $M$ & 78 & $42.38 \pm 15.69$ & 44 & 0.48 & -4.35 & 0.536 \\
\hline $3-1$ & - & w;255B/CyO; frulR/+ & $\mathrm{F}$ & 79 & $73.75 \pm 13.13$ & 76 & ------- & ------- & ------- \\
\hline $3-1$ & A & w; 255B/CyO; frulR/+ & $\mathrm{F}$ & 86 & $57.88 \pm 14.28$ & 62 & -21.51 & -18.42 & 0 \\
\hline $3-1$ & $\mathrm{~L}$ & w; 255B/CyO; frulR/+ & $\mathrm{F}$ & 101 & $59.07 \pm 13.13$ & 62 & -19.9 & -18.42 & 0 \\
\hline $6-1$ & - & $w / Y ; 255 B /+;+/+$ & $M$ & $12 \mid$ & $62.33 \pm 18.12$ & 68 & --..-- & --.-- & ------- \\
\hline $6-1$ & $A$ & $w / Y ; 255 B /+;+/+$ & $M$ & 117 & $57.09 \pm 22.64$ & 66 & -8.4 & -2.94 & 0.165 \\
\hline $6-1$ & $\mathrm{~L}$ & $w / Y ; 255 B /+;+/+$ & $M$ & 119 & $62.57 \pm 16.22$ & 68 & 0.39 & 0 & 0.478 \\
\hline $6-1$ & - & $w / w / I / 8 ; 255 B /+;+/+$ & $\mathrm{F}$ & 123 & $75.95 \pm 9.37$ & 78 & -------- & -------- & -------- \\
\hline $6-1$ & A & $w / w / I / 8 ; 255 B /+;+/+$ & $\mathrm{F}$ & 122 & $71.05 \pm 13.36$ & 74 & -6.45 & -5.13 & 7.97E-06 \\
\hline $6-1$ & $\mathrm{~L}$ & $w / w / I / 8 ; 255 B /+;+/+$ & $\mathrm{F}$ & 124 & $69.02 \pm 12.88$ & 74 & -9.13 & -5.13 & 7.69E-07 \\
\hline \multicolumn{10}{|l|}{$\operatorname{Exp} 3$} \\
\hline $7-1$ & - & $w / Y ; 255 B /+;+$ & $M$ & 124 & $72.03 \pm 18.30$ & 75 & ------- & ------- & -------- \\
\hline $7-1$ & $\mathrm{~A}$ & $w / Y ; 255 B /+;+$ & $M$ & 123 & $74.18 \pm 20.23$ & 80 & 2.98 & 6.67 & 0.151 \\
\hline $7-1$ & $\mathrm{~L}$ & $w / Y ; 255 B /+;+$ & $M$ & 123 & $71.69 \pm 23.51$ & 80 & -0.47 & 6.67 & 0.171 \\
\hline $7-1$ & - & $w /+; 255 B /+;+$ & $\mathrm{F}$ & 124 & $85.39 \pm 25.75$ & 92 & -------- & -------- & -------- \\
\hline $7-1$ & A & $w /+; 255 B /+;+$ & $\mathrm{F}$ & 118 & $83.03 \pm 25.60$ & 90 & -2.76 & -2.17 & 0.043 \\
\hline $7-1$ & $\mathrm{~L}$ & $w /+; 255 B /+;+$ & $\mathrm{F}$ & 119 & $91.78 \pm 19.02$ & 96 & 7.49 & 4.35 & 0.213 \\
\hline$|-||R|$ & - & y w sxl/Y; 255B/+; + & $M$ & 25 & $85.12 \pm 14.30$ & 88 & -------- & ------- & ------- \\
\hline$|-| I R I$ & A & y w sxl/Y; 255B/+; + & $M$ & 45 & $85.51 \pm 19.71$ & 94 & 0.46 & 6.82 & 0.314 \\
\hline$|-||R|$ & $\mathrm{L}$ & y w sxl/Y; 255B/+; + & $M$ & 76 & $85.13 \pm 13.94$ & 84 & 0.01 & -4.55 & 0.635 \\
\hline$|-| I R I$ & - & y w sxl/w; 255B/+; + & $\mathrm{F}$ & 49 & $70.45 \pm 31.82$ & 82 & --.- & --.--- & ------- \\
\hline$|-||R|$ & A & y w sxl/w; 255B/+; + & $\mathrm{F}$ & 72 & $57.39 \pm 35.23$ & 76 & -18.54 & -7.32 & 0.011 \\
\hline$|-||R|$ & $\mathrm{L}$ & y w sxl/w; 255B/+; + & $\mathrm{F}$ & 122 & $83.31 \pm 23.54$ & 86 & 18.26 & 4.88 & 0.005 \\
\hline I-II R2 & - & y w sxl/Y; 255B/+; + & $M$ & 67 & $91.22 \pm 21.02$ & 98 & ------- & ------- & -------- \\
\hline I-II R2 & A & y w sxl/Y; 255B/+; + & $M$ & 75 & $75.55 \pm 22.66$ & 80 & -17.19 & -18.37 & 2.30E-09 \\
\hline I-II R2 & $\mathrm{L}$ & y w sxl/Y; 255B/+; + & $M$ & 96 & $86.31 \pm 27.48$ & 98 & -5.38 & 0 & 0.965 \\
\hline I-II R2 & - & y w sxl/w; 255B/+; + & $\mathrm{F}$ & 69 & $89.45 \pm 25.26$ & 92 & ----- & --.--- & -------- \\
\hline I-II R2 & A & y w sxl/w; 255B/+; + & $\mathrm{F}$ & 74 & $87.32 \pm 18.37$ & 88 & -2.38 & -4.35 & 0.064 \\
\hline$I-I \mid R 2$ & $\mathrm{~L}$ & y w sxl/w; 255B/+; + & $\mathrm{F}$ & 121 & $92.58 \pm 17.93$ & 96 & 3.5 & 4.35 & 0.593 \\
\hline $11-7$ & - & y w sxl/Y; +; + & $M$ & 119 & $92.76 \pm 15.18$ & 98 & ------- & ----- & ------ \\
\hline $11-7$ & - & y w sxll+; +; + & $\mathrm{F}$ & 120 & $94.48 \pm 18.26$ & 98 & -------- & -------- & -------- \\
\hline $10-1$ & - & $w / Y ; 255 B / t r a ;+$ & $M$ & 124 & $81.47 \pm 14.12$ & 82 & -------- & -------- & -------- \\
\hline $10-1$ & A & w/Y; 255B/tra; + & $M$ & 122 & $74.48 \pm 13.90$ & 76 & -8.58 & -7.32 & 2.26E-04 \\
\hline $10-1$ & $\mathrm{~L}$ & w/Y; 255B/tra; + & $M$ & 124 & $79.71 \pm 16.97$ & 84 & -2.16 & 2.44 & 0.293 \\
\hline $10-1$ & - & w; 255B/tra; + & $\mathrm{F}$ & 127 & $93.40 \pm 15.91$ & 96 & ----- & --.-- & ------- \\
\hline $10-1$ & A & w; 255B/tra; + & $\mathrm{F}$ & 123 & $91.38 \pm 18.93$ & 98 & -2.16 & 2.08 & 0.81 \\
\hline $10-1$ & $\mathrm{~L}$ & w; 255B/tra; + & $\mathrm{F}$ & 126 & $90.24 \pm 22.04$ & 96 & -3.39 & 0 & 0.732 \\
\hline$|5-|$ & - & w/Y; 255B/tra2; + & $M$ & 125 & $73.44 \pm 16.92$ & 76 & ------- & ------- & ------ \\
\hline $15-1$ & A & w/Y; 255B/tra2; + & $M$ & 124 & $75.61 \pm 15.06$ & 76 & 2.96 & 0 & 0.469 \\
\hline $15-1$ & $\mathrm{~L}$ & $w / Y ; 255 B / t r a 2 ;+$ & $M$ & 125 & $74.64 \pm 21.79$ & 78 & 1.63 & 2.63 & 0.039 \\
\hline $15-1$ & - & w; $255 \mathrm{~B} /$ tra2; + & $\mathrm{F}$ & 124 & $78.58 \pm 25.27$ & 85 & -------- & ------- & -------- \\
\hline$|5-|$ & A & w;255B/tra2; + & $\mathrm{F}$ & 125 & $77.47 \pm 27.00$ & 86 & $-|.4|$ & 1.18 & 0.789 \\
\hline $15-1$ & $\mathrm{~L}$ & w;255B/tra2; + & $\mathrm{F}$ & 125 & $79.81 \pm 17.45$ & 82 & 1.56 & -3.53 & 0.144 \\
\hline $9-1$ & - & $w / Y ; 255 B /+; d s x F /+$ & $M$ & 74 & $83.35 \pm 22.08$ & 88 & -------- & -------- & --------- \\
\hline $9-1$ & A & $w / Y ; 255 B /+; d s x F /+$ & $M$ & 100 & $34.20 \pm 9.96$ & 32 & -58.97 & -63.64 & 0 \\
\hline $9-1$ & $\mathrm{~L}$ & $w / Y ; 255 B /+; d s x F /+$ & $M$ & 0 & ------- & --- & ------- & ------- & -------- \\
\hline $9-1$ & - & $w ; 255 B /+; d s x F /+$ & $\mathrm{F}$ & 73 & $87.53 \pm 19.27$ & 90 & -------- & ------- & -------- \\
\hline $9-1$ & A & $w ; 255 B /+; d s x F /+$ & $\mathrm{F}$ & 98 & $52.00 \pm|3.5|$ & 52 & -40.59 & -42.22 & 0 \\
\hline $9-1$ & $\mathrm{~L}$ & w; $255 B /+; d s x F /+$ & $\mathrm{F}$ & 24 & $67.33 \pm 26.60$ & 78 & -23.08 & -13.33 & I.87E-04 \\
\hline$|4-|$ & - & $w / Y ; 255 B /++d s x /+$ & $M$ & 124 & $68.73 \pm 21.07$ & 72 & -------- & -------- & -------- \\
\hline $\mid 4-1$ & A & $w / Y ; 255 B /+; d s x /+$ & $M$ & 124 & $70.53 \pm 17.57$ & 72 & 2.63 & 0 & 0.88 \\
\hline $14-1$ & $\mathrm{~L}$ & $w / Y ; 255 B /+; d s x /+$ & $M$ & 5 & $46.00 \pm 35.30$ & 50 & -33.07 & -30.56 & 0.27 \\
\hline $14-1$ & - & $w ; 255 B /+; d s x /+$ & $\mathrm{F}$ & 123 & $65.37 \pm 28.78$ & 76 & ------- & ------- & ------- \\
\hline$|4-|$ & $A$ & w; $255 B /+; d s x /+$ & $\mathrm{F}$ & 123 & $65.56 \pm 30.01$ & 76 & 0.3 & 0 & 0.763 \\
\hline$|4-|$ & $\mathrm{L}$ & $w ; 255 B /+; d s x /+$ & $\mathrm{F}$ & 123 & $73.06 \pm|7.5|$ & 74 & 11.77 & -2.63 & 0.735 \\
\hline
\end{tabular}

a Mean life span, days $+/$ - SD. 

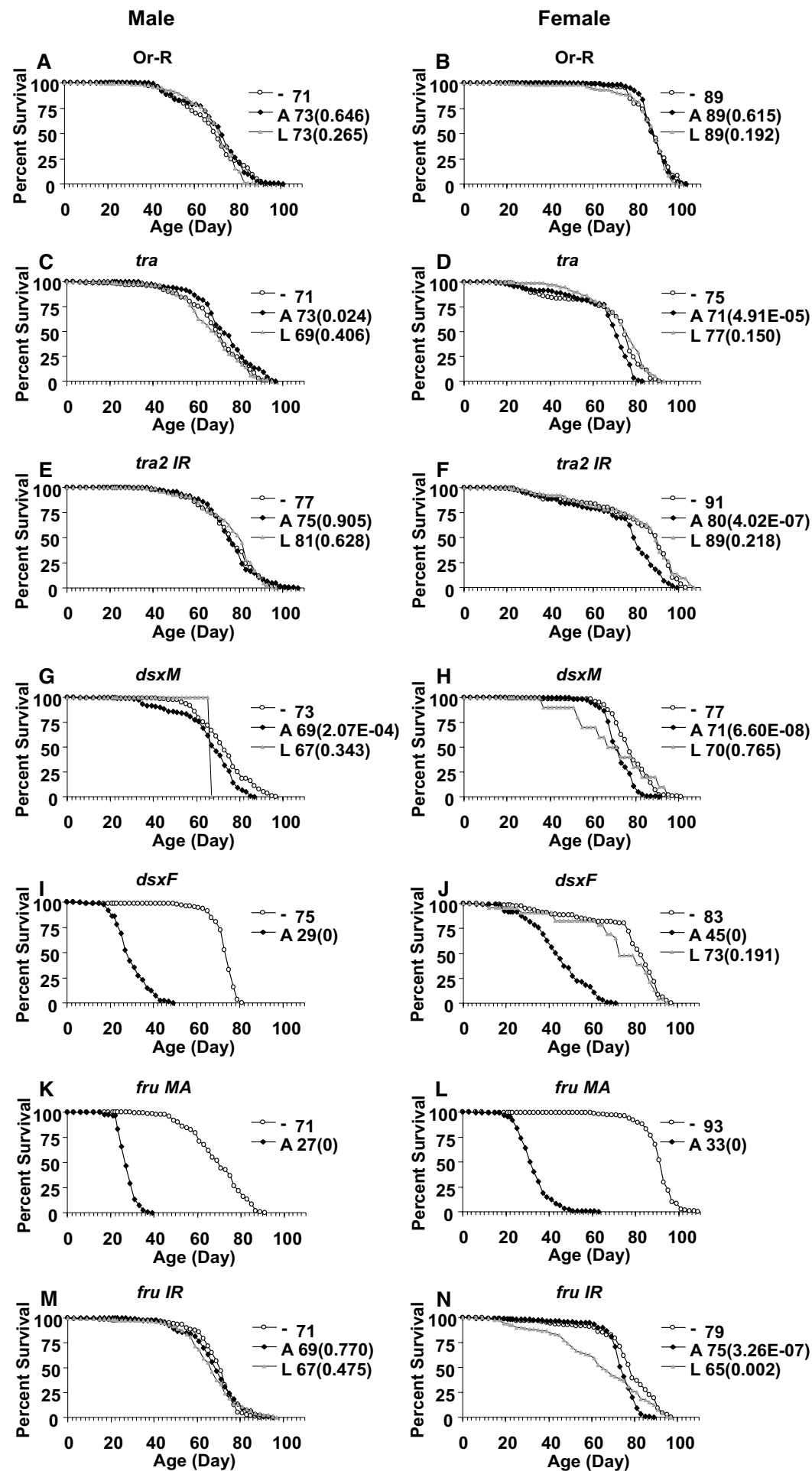

\section{Figure 3}

Effect of sex differentiation pathway gene mis-expression on survival of male and female adult flies. Sexual differentiation pathway genes or RNAi constructs were over-expressed either during larval development ("L"; gray triangles) or in adults ("A"; solid squares). Open circles represent the no-drug control ("-"). Survival curves are plotted as a function of adult age in days. Median life span and $p$ value for log rank test are indicated in parentheses for each cohort. (A, B) Control flies (progeny of driver crossed to Or-R wild type). (C, D) tra. (E, F) tra2-IR. (G, H) dsxM. (I, J) dsxF. (K, L) fruMA. (M, N) fru-IR. 


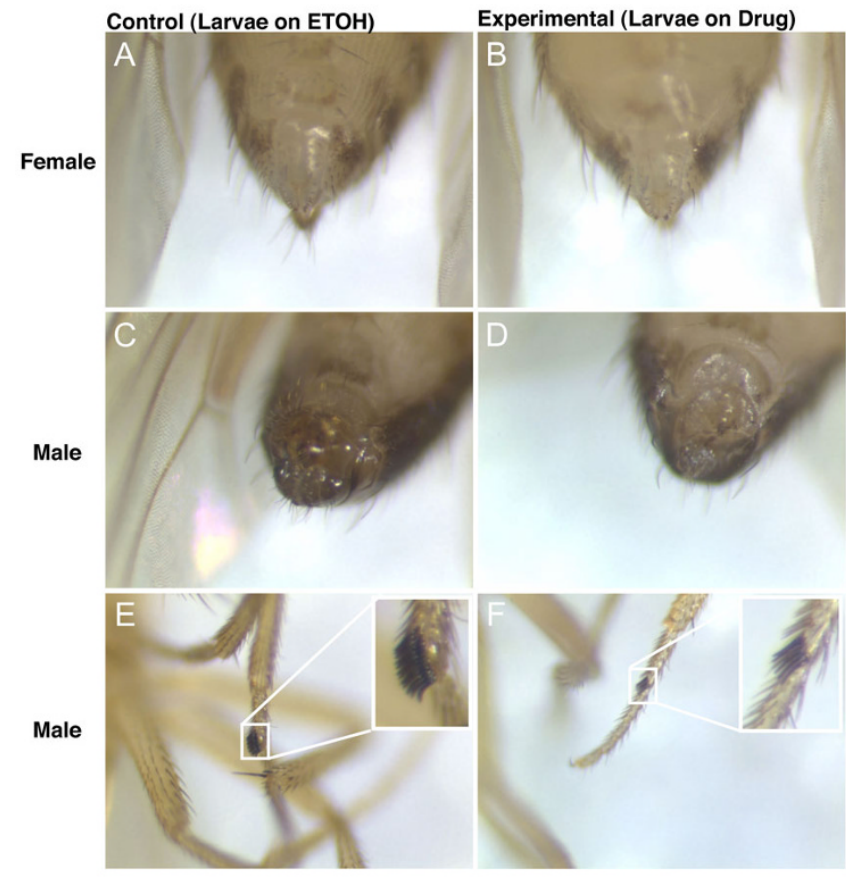

Figure 4

Effect of tra over-expression during development on sexual differentiation of adults. UAS-tra males were crossed to GS255B virgins, cultured on food with drug (+RU486) to drive the over-expression of tra during development, or cultured on food with ethanol as the control, as indicated. Pictures were taken at the magnification of I00X. (A, B) Female genitalia. (C, D) Male genitalia. (E, F) Male sex comb.

sion of fruMA in adults greatly reduced both male and female mean life span. However, expression of an RNAi construct specific for fruMA during development or in adults significantly decreased female life span, but did not give a consistent change in male life span. The reason for this effect of fruMA-RNAi in females is not yet clear. In females, fru P1 transcripts are produced, but not translated [24]. Possibly the fruMA-RNAi could still function through fru P1 transcripts to affect female life span, or could function through targets other than fru.

Taken together with life span results of other assayed genes, our data show little correspondence between the sex-specific mode of action of sexual differentiation genes in development and sex-specific effects of their overexpression or knockdown on adult lifespan. For example, adult-specific over-expression of fruMA and $d s x F$ shortened life span to a similar extent in males and females. This may be because the sex-specificity of these factors is created during development by means of sex-specific expression, and in our experiments we are forcing expression in each sex, thereby eliminating sex-specificity of action. One possible exception is the developmental lethality caused by over-expression of $d s x F$ during larval development, which was complete in males, where it is not normally expressed, and only partial in females, where it is normally present.

Sexual differentiation genes play important roles in germ cell development [20-22]. Therefore, one way that sexual differentiation genes might regulate life span is by modulating germ cell development. Sexual differentiation genes also regulate behavior; for example, fru is essential to generate several male-specific behaviors, including male courtship behavior [43]. Although the role of sexual differentiation genes on behavior has not been examined specifically in the adult stage, one way sexual differentiation genes might conceivably affect life span is by altering costly or beneficial behavior patterns in adults. Another promising direction for future experiments will be to look at the interactions between sexual differentiation genes and the pathways and interventions that are known to regulate life span in a sex-specific manner, such as the insulin/IGF1-like signaling (IIS) pathway and dietary restriction (DR) $[8,10,11]$.

\section{Conclusion}

The data demonstrate that manipulation of sexual differentiation gene expression specifically in the adult, after morphological sexual differentiation is complete, is able to affect life span. In addition, by manipulating gene expression during development, it was possible to significantly alter morphological sexual differentiation without a significant effect on adult life span. The data demonstrate that manipulation of sexual differentiation pathway genes either during development or in adults can affect adult life span, sometimes with sex-specific effects, and suggest it should be possible in the future to investigate how the sexual differentiation pathway interacts with specific life span regulatory genes to produce sex-specific differences in longevity.

\section{Competing interests}

The authors declare that they have no competing interests.

\section{Authors' contributions}

DF conducted life span assay of tudor mutant offspring. JS and GL conducted experiments with gene over-expression and RNAi. JS performed statistical analyses, with help from DF and GL. JT conceived of and supervised the project, and JT and JS wrote the paper. All authors have read and approved the final manuscript.

\section{Acknowledgements}

We thank Michelle Arbeitman and Aaron Tarone for comments. This work was supported by a grant from the Department of Health and Human Services to JT (ROIAG0I I833). 


\section{References}

I. Kirkwood TB: Understanding the odd science of aging. Cell 2005, I 20(4):437-447.

2. Kirkwood TBL: The disposable soma theory of aging. In Genetic effects on aging II Edited by: Harrison DE. Caldwell, NJ: Telford press.; 1990.

3. Westendorp RG, Kirkwood TB: Human longevity at the cost of reproductive success. Nature 1998, 396(67 I 3):743-746.

4. Ligtenberg $\mathrm{T}$, Brand $\mathrm{H}$ : Longevity--does family size matter? Nature 1999, 399(6736):522.

5. Gavrilova NS, Gavrilov LA, Semyonova VG, Evdokushkina GN: Does exceptional human longevity come with a high cost of infertility? Testing the evolutionary theories of aging. Ann N Y Acad Sci 2004, 1019:513-517.

6. Hsin H, Kenyon C: Signals from the reproductive system regulate the lifespan of C. elegans. Nature 1999, 399(6734):362-366

7. Berman JR, Kenyon C: Germ-cell loss extends C. elegans life span through regulation of DAF-I 6 by kri-I and lipophilichormone signaling. Cell 2006, I24(5): I055-1068.

8. Toivonen JM, Partridge L: Endocrine regulation of ageing and reproduction in Drosophila. Mol Cell Endocrinol 2008, 299(I):39-50.

9. Flatt $\mathrm{T}$, Kawecki TJ: Juvenile hormone as a regulator of the trade-off between reproduction and life span in Drosophila melanogaster. Evolution 2007, 6 I (8): 1980-1991.

10. Tower J: Sex-specific regulation of aging and apoptosis. Mech Ageing Dev 2006, I 27(9):705-7I8.

II. Burger JM, Promislow DE: Sex-specific effects of interventions that extend fly life span. Sci Aging Knowledge Environ 2004, 2004(28):pe30.

12. Magwere T, Chapman T, Partridge L: Sex differences in the effect of dietary restriction on life span and mortality rates in female and male Drosophila melanogaster. J Gerontol A Biol Sci Med Sci 2004, 59(I):3-9.

13. Shen J, Curtis C, Tavaré S, Tower J: A screen of apoptosis and senescence regulatory genes for life span effects when overexpressed in Drosophila. Impact Aging 2009, I(2):|9|-21।.

14. Bauer JH, Poon PC, Glatt-Deeley H, Abrams JM, Helfand SL: Neuronal expression of p53 dominant-negative proteins in adult Drosophila melanogaster extends life span. Curr Biol 2005, I 5(22):2063-2068.

15. Tower J, Arbeitman M: The genetics of gender and life span. J Biol 2009, 8(4):38.

16. Flatt T, Min KJ, D'Alterio C, Villa-Cuesta E, Cumbers J, Lehmann R, Jones DL, Tatar M: Drosophila germ-line modulation of insulin signaling and lifespan. Proc Natl Acad Sci USA 2008, I 05(I 7):6368-6373

17. Thomson T, Lasko P: Tudor and its domains: germ cell formation from a Tudor perspective. Cell Res 2005, I 5(4):28I-29I.

18. Barnes Al, Boone JM, Jacobson J, Partridge L, Chapman T: No extension of lifespan by ablation of germ line in Drosophila . Proc Biol Sci 2006, 273( I 589):939-947.

19. Camara N, Whitworth C, Van Doren M: The creation of sexual dimorphism in the Drosophila soma. Curr Top Dev Biol 2008, 83:65-107.

20. Oliver B: Genetic control of germline sexual dimorphism in Drosophila . Int Rev Cytol 2002, 2 I 9: I-60.

21. Gilboa L, Lehmann R: How different is Venus from Mars? The genetics of germ-line stem cells in Drosophila females and males. Development 2004, I 3 I (20):4895-4905.

22. Casper A, Van Doren M: The control of sexual identity in the Drosophila germline. Development 2006, I 33( I 5):2783-279|.

23. Goldman TD, Arbeitman MN: Genomic and functional studies of Drosophila sex hierarchy regulated gene expression in adult head and nervous system tissues. PLoS Genet 2007, 3(I I):e2 I6.

24. Song HJ, Billeter JC, Reynaud E, Carlo T, Spana EP, Perrimon N, Goodwin SF, Baker BS, Taylor BJ: The fruitless gene is required for the proper formation of axonal tracts in the embryonic central nervous system of Drosophila . Genetics 2002, I 62(4): I703-1724.

25. Manoli DS, Baker BS: Median bundle neurons coordinate behaviours during Drosophila male courtship. Nature 2004, 430(6999):564-569.
26. Lee G, Hall JC, Park JH: Doublesex gene expression in the central nervous system of Drosophila melanogaster. J Neurogenet 2002, I 6(4):229-248.

27. Sanchez L, Gorfinkiel N, Guerrero I: Sex determination genes control the development of the Drosophila genital disc, modulating the response to Hedgehog, Wingless and Decapentaplegic signals. Development 200I, I 28(7): I033-I043.

28. Ferveur JF, Greenspan RJ: Courtship behavior of brain mosaics in Drosophila. J Neurogenet 1998, I2(4):205-226.

29. Thibault ST, Singer MA, Miyazaki WY, Milash B, Dompe NA, Singh CM, Buchholz R, Demsky M, Fawcett R, Francis-Lang HL, et al:: A complementary transposon tool kit for Drosophila melanogaster using $\mathbf{P}$ and piggyBac. Nat Genet 2004, 36(3):283-287.

30. Schupbach T, Wieschaus E: Maternal-effect mutations altering the anterior-posterior pattern of the Drosophila embryo. Rouxs Arch Dev Biol 1986, 195:302-317.

31. Ford D, Hoe N, Landis GN, Tozer K, Luu A, Bhole D, Badrinath A, Tower J: Alteration of Drosophila life span using conditional, tissue-specific expression of transgenes triggered by doxycycline or RU486/Mifepristone. Exp Gerontol 2007, 42(6):483-497.

32. Ren C, Webster P, Finkel SE, Tower J: Increased internal and external bacterial load during Drosophila aging without lifespan trade-off. Cell Metab 2007, 6(2):|44-I52.

33. O'Neill SL, Giordano R, Colbert AM, Karr TL, Robertson HM: I6S rRNA phylogenetic analysis of the bacterial endosymbionts associated with cytoplasmic incompatibility in insects. Proc Natl Acad Sci USA 1992, 89(7):2699-2702.

34. Pletcher SD: Model fitting and hypothesis testing for age-specific mortality data. J Evol Biol 1999, I 2:430-439.

35. Moran NA, McCutcheon JP, Nakabachi A: Genomics and evolution of heritable bacterial symbionts. Annu Rev Genet 2008 , 42: $165-190$.

36. Fry AJ, Palmer MR, Rand DM: Variable fitness effects of Wolbachia infection in Drosophila melanogaster. Heredity 2004, 93(4):379-389.

37. Jursnich VA, Burtis KC: A positive role in differentiation for the male doublesex protein of Drosophila. Dev Biol 1993, I 55( I):235-249.

38. Allikian MJ, Deckert-Cruz D, Rose MR, Landis GN, Tower J: Doxycycline-induced expression of sense and inverted-repeat constructs modulates phosphogluconate mutase (Pgm) gene expression in adult Drosophila melanogaster. Genome Biol 2002, 3(5):research002I.

39. Ren C, Finkel SE, Tower J: Conditional inhibition of autophagy genes in adult Drosophila impairs immunity without compromising longevity. Exp Gerontol 2009, 44(3):228-235.

40. Smith JM: The effects of temperature and of egg-laying on the longevity of Drosophila subobscura. Journal of Experimental Biology 1958, 35:832-842.

4I. Yamawaki TM, Arantes-Oliveira N, Berman JR, Zhang P, Kenyon C: Distinct activities of the germline and somatic reproductive tissues in the regulation of Caenorhabditis elegans ' longevity. Genetics 2008, I 78(I):513-526.

42. Gatti S, Ferveur JF, Martin JR: Genetic identification of neurons controlling a sexually dimorphic behaviour. Curr Biol 2000, I 0(I I):667-670.

43. Demir E, Dickson BJ: fruitless splicing specifies male courtship behavior in Drosophila. Cell 2005, I 2 I (5):785-794.

\section{Pre-publication history}

The pre-publication history for this paper can be accessed here:

http://www.biomedcentral.com/1471-2318/9/56/prepub 